\title{
A TOPOLOGICAL CHARACTERIZATION OF REAL ALGEBRAIC VARIETIES
}

BY SELMAN AKBULUT AND HENRY C. KING

We show that if a smooth locally conelike stratified set admits a certain kind of topological resolution then it is homeomorphic to a real algebraic set, i.e. zeros of polynomial functions (this generalizes $\left[\mathbf{A K}_{\mathbf{1}}\right],\left[\mathbf{A K}_{\mathbf{2}}\right]$ ). We expect that the algebraic resolution of singularities [H] implies that every algebraic set admits such a topological resolution, hence it is reasonable to suspect that we have a complete topological characterization of real algebraic sets.

Examples of some stratified sets admitting such a topological resolution are spaces which we call $A_{k}$-spaces, $k=0,1,2, \cdots$. We define $A_{k}$-spaces inductively by saying that $A_{0}$-spaces are smooth compact manifolds, and an $A_{k}$ space is a compact smooth stratified set $X$ with a trivialization of a neighborhood of each stratum $X_{i}, h_{i}: X_{i} \times \operatorname{cone}\left(\Sigma_{i}\right) \rightarrow X$ where $\Sigma_{i}$ is an $A_{k-1}$-space which bounds a compact $A_{k-1}$-space with boundary ( $h_{i}$ required to be compatible with the trivializations of neighborhoods of the strata of $\Sigma_{i}$ ).

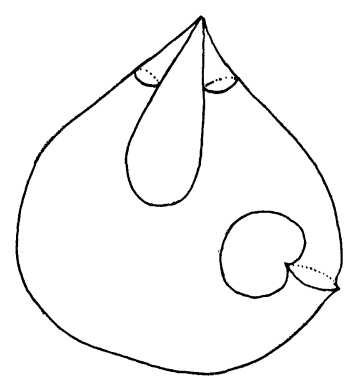

an $A_{1}$-space

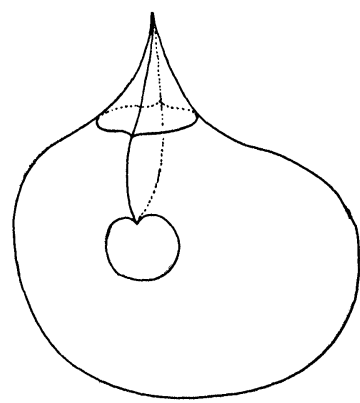

an $A_{2}$-space

The topological resolution of an $A_{k}$-space $X$ is obtained by a sequence of 'blow ups' as follows: take a lowest dimensional stratum $X_{i}$ (the 'center' of the 'blow ups') with trivialization $h_{i}: X_{i} \times \operatorname{cone}\left(\Sigma_{i}\right) \rightarrow X$ and replace $h_{i}\left(X_{i} \times \operatorname{cone}\left(\Sigma_{i}\right)\right)$ by $X_{i} \times W_{i}$ where $W_{i}$ is a compact $A_{k-1}$-space which $\Sigma_{i}$

Received by the editors September 4, 1979.

AMS (MOS) subject classifications (1970). Primary 57C05, 14A10, 14E15. manifolds.

Key words and phrases. Algebraic sets, resolutions, $A_{k}$-spaces, stratifications, P.L. 
bounds. We have a map from the new space to $X$ which is the identity outside image $\left(h_{i}\right)$ and which collapses $X_{i} \times S_{i}$ to $X_{i} \times *$ where $S_{i}$ is a spine of $W_{i}$ and * is the vertex of cone $\left(\Sigma_{i}\right)$. After a finite number of such blow ups we obtain a smooth manifold $\tilde{X}$ and a 'resolution' $\widetilde{X}=Z_{n} \rightarrow Z_{n-1} \rightarrow \cdots \rightarrow Z_{0}=X$. We say $X$ is an $A$-space if it is an $A_{k}$-space for some $k$. In particular we prove:

THEOREM. The interior of any compact A-space is homeomorphic to a real algebraic set. Furthermore the natural stratification on this algebraic set coincides with the stratification of the A-structure.

One of the reasons $A$-spaces are of interest is that Akbulut and Taylor have shown that any compact P.L. manifold has the structure of an $A$-space [AT]

Corollary. The interior of any compact P.L. manifold is P.L homeomorphic to a real algebraic set.

SKETCH OF PROOF. Take a resolution of a compact $A_{k}$-space $X: Z_{n} \rightarrow$ $Z_{n-1} \rightarrow \cdots \rightarrow Z_{0}=X$ where $Z_{n}$ is a smooth compact manifold. Each $Z_{i+1}$ $\rightarrow Z_{i}$ has a certain 'center' a smooth manifold $X_{i} \subset Z_{i}$ along which a topological 'blow up' occurs. We construct a tower of nonsingular varieties $V_{n} \rightarrow V_{n-1}$ $\longrightarrow \cdots \rightarrow V_{0}=\mathbf{R}^{n}$ with $X_{i} \subset V_{i}$ as a nonsingular subvariety and imbeddings $Z_{i} \subset V_{i}$ which commute with projections (i.e. all the above maps given by arrows) and are in some sense 'stable' over the projections.

This means that if $Z_{n}$ is moved by a small isotopy in $V_{n}$ the image of $Z_{n}$ under the composite projection $\pi: V_{n} \rightarrow V_{0}$ is isotopic to $X$. We then approximate the submanifold $Z_{n} \subset V_{n}$ by an algebraic set $Q$; and then 'blow down' $Q$ algebraically to an algebraic set $V$ which is homeomorphic to $\pi(Q)$, which is in turn homeomorphic to $X$.

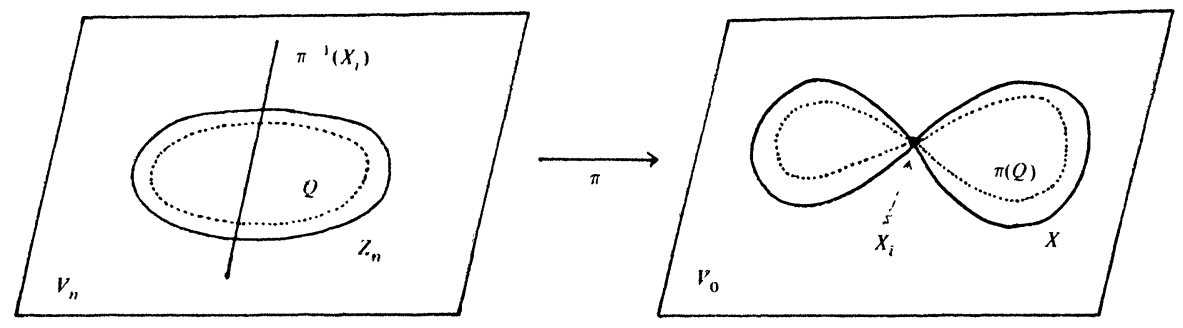

Each $V_{i+1}$ and the projection $V_{i+1} \stackrel{\pi_{i}}{\longrightarrow} V_{i}$ is obtained by a certain algebraic 'multiblowing-up' process from $V_{i}$ along $X_{i} . X_{i}$ is a lowest dimensional stratum of $Z_{i}$. Given $Z_{i} \subset V_{i}$ the imbedding $Z_{i+1} \subset V_{i+1}$ is obtained roughly as follows: Let $h_{i}: X_{i} \times \operatorname{cone}\left(\Sigma_{i}\right) \rightarrow Z_{i}$ be the neighborhood trivialization of $X_{i}$, and $W_{i}$ a compact $A_{k}$-space which $\Sigma_{i}$ bounds; imbed $X_{i} \times W_{i}$ into $V_{i+1}$ so that 
(a) $X_{i} \times W_{i}$ is transverse to $\pi_{i}^{-1}\left(X_{i}\right)$,

(b) $\pi_{i}^{-1}\left(X_{i}\right) \cap\left(X_{i} \times W_{i}\right)=X_{i} \times\left(\right.$ a spine of $\left.W_{i}\right)$,

(c) $\pi_{i}\left(X_{i} \times W_{i}\right) \approx h_{i}\left(X_{i} \times \operatorname{cone}\left(\Sigma_{i}\right)\right)$.

Then extend this imbedding to an imbedding of $Z_{i+1}$ into $V_{i+1}$ by simply lifting the imbedding $Z_{i}$-image $\left(h_{i}\right)\left(\approx Z_{i+1}-X_{i} \times W_{i}\right)$ to $V_{i+1}$ via $\pi_{i}$ so that $\pi_{i}\left(Z_{i+1}\right) \approx Z_{i}$. In particular, along the way we prove that the $A_{k}$-space $\Sigma_{i}$, which bounds, necessarily has to bound an $A_{k}$-space $W_{i}$, which has a spine consisting of transversally intersecting codimension one $A_{k}$-subspaces without boundaries. Choosing such $W_{i}$ 's enables us to show (a), (b), (c). Details are long and geometric in nature, they will appear in $\left[\mathbf{A K}_{\mathbf{3}}\right]$. The proof applies to spaces more general than $A$-spaces, which leads us to believe that a satisfactory topological classification theorem for real algebraic sets is within reach.

\section{REFERENCES}

$\left[\mathrm{AK}_{1}\right]$ S. Akbulut and H. C. King, Real algebraic variety structures on P.L. manifolds, Bull. Amer. Math. Soc. 83 (1977), 281-282.

$\left[\mathrm{AK}_{2}\right]-$ The topology of real algebraic sets with isolated singularities, Ann. of Math. (to appear).

[AK $\left.\mathbf{A K}_{3}\right] \longrightarrow$ Real algebraic structures on topological spaces (to appear).

[AT] S. Akbulut and L. Taylor, A topological resolution theorem, Bull. Amer. Math. Soc. N.S. 2 (1980), 174-176.

[H] H. Hironaka, Resolution of singularities of an algebraic variety over a field of characteristic zero, Ann of Math. 79 (1964), 109-326.

DEPARTMENT OF MATHEMATICS, RUTGERS UNIVERSITY, NEW BRUNSWICK, NEW JERSEY 08903

DEPARTMENT OF MATHEMATICS, UNIVERSITY OF MARYLAND, COLLEGE PARK, MARYLAND 20742 